\title{
FREEFORM FABRICATION OF POLYMER-MATRIX COMPOSITE STRUCTURES*
}

\author{
Stephen G. Kaufman \\ Intelligent Systems and Robotics Center \\ Sandia National Laboratories
}

\author{
Barry L. Spletzer \\ Intelligent Systems and Robotics Center \\ Sandia National Laboratories
}

\author{
Tommy L. Guess \\ Manufacturing Technologies Center \\ Sandia National Laboratories
}

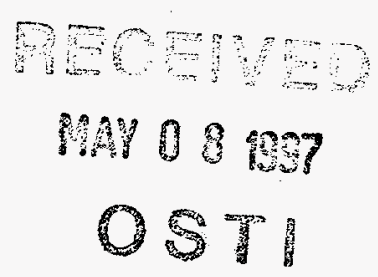

is built up layer by layer, using a computer-controlled laser to selectively cure epoxy resin. To produce a new shape, only the software controlling the curing is changed; no changes to the hardware are necessary. However, stereolithography can only build structures composed of pure resin, or resin containing chopped (non-continuous) fiber. Another example of rapid prototyping is laser sintering; in this technique, the laser energy bonds powdered metal and/or ceramic. Given the success of these technologies, it is natural to seek a method of rapid prototyping for CFPM composites, so that the domain of rapid prototyping is extended to these high-modulus, low-weight materials. Such a method would be able to produce shapes of high complexity, not subject to the shape constraints imposed by the need for molds; furthermore, the only change to the system needed to produce a new shape would be to the software, which would be automatically generated from an electronic model of the shape. Such a method would have the dual advantages of being able to produce shapes currently producible only with great difficulty, and at lower cost since mandrels need not be made.

We have developed a method of producing structures composed of CFPM composites that does not require a mandrel, and is therefore not subject to these constraints on producible shapes. This allows shapes to be produced that are difficult to make by existing methods. For example, a cylinder-like object, with a square cross-section in the middle and circular crosssection at the ends, can be made with our method, but filament winding would require a destructively removable mandrel. Furthermore, the method is implemented with programmable devices, allowing automatic programming of the system to produce a shape from an electronic model of it. The method is the basis of a CFPM rapid prototyping system as envisioned in the previous paragraph. An example is stereolithography, in which the shape

* This work was supported by Sandia National Laboratories under DOE contract DE-AC04-94AL85000, and by the Laboratory Directed Research and Development Office of Sandia National Laboratories. 
We have demonstrated that our method has the capability to:

1. Produce composite parts of the desired shape under automatic control (to date, we have produced right circular cylinders of various thicknesses);

2. Produce satisfactorily consolidated composites using preconsolidated or commingled thermoplastic resin with graphite or Kevlar fibers;

3. Produce satisfactory parts of significant thickness (14 layers have been demonstrated).

The quality of the parts we have produced is comparable to that of parts produced using filament winding on a mandrel.

The remainder of this paper is divided into two major sections: a summary of existing fabrication methods; and a description of our system. The conclusion describes our on-going work.

\section{FABRICATION METHODS FOR COMPOSITES}

Fabrication methods for composite structures fall into six basic categories: layup, resin transfer molding, filament winding, fiber placement, pultrusion, and braiding/weaving. We describe each in turn. The boundaries between methods are often fuzzy, as the reader will observe. More complete descriptions are available elsewhere, for example, (Harper, 1992).

\section{Background and Terminology}

A composite material, as its name implies, consists of two or more distinct materials. The composite exhibits the best properties of the constituent materials. Wellknown examples are bricks made of mud and straw, and structures made of concrete and reinforcing bars. Composites of ceramic and metal have recently become available.

The composites of interest in this work consist of a high-modulus fiber and a polymer binder. The non-fibrous material is called the matrix; examples are polymers such as epoxy resins and thermoplastics such as nylon. The types of matrix are subdivided into thermosets and thermoplastics. Thermosetting materials are chemically and irreversibly altered during the cure process, which often involves the application of heat (in some cases, the heat is the exotherm of the curing reaction). Thermoplastics simply melt when heated; they may be remelted a number of times, though they degrade after a number of heating/cooling cycles.

These materials are available in a number of forms. The fiber may be separate from the matrix material, or it may be already impregnated with it. The latter form is called prepreg. Thermoplastics, which are solid at room temperature, may be commingled, or woven, with the fibers, or preconsolidated, where they are already melted together with the fiber. Preconsolidated and prepreg materials are often sold as rolls of flat tape.

\section{Layup}

In this process, fiber and matrix material are placed in a mold. The fiber and matrix may be applied separately or simultaneously. Once the placement is complete, the resin is cured. This can be at room temperature and pressure, but better results are obtained when both quantities are elevated in an autoclave or press.

In hand layup, fiber mats are placed, resin is sprayed or painted on, and is pressed into the fiber with rollers or squegees. Then the material cures at room temperature.

Fiber and resin can be combined before layup in several ways. In spray layup, fiber is cut and combined with resin in a spray gun, which is then used to coat the mold. Prepreg materials can also be used; when they are, automated layup becomes easier, in the form of automatic tape layup. The system described by Olsen and Craig (1993) consists of a robot mounted prepreg tape dispenser. This dispensing head is capable of cutting the tape, restarting the dispensing process, and applying pressure at the point of application. It differs from filament winding in this ability to stop dispensing tape (by cutting) and restart at a different point.

\section{Resin Transfer Molding}

The hallmark of resin-transfer molding (RTM) is the injection of matrix resin into a closed mold which contains the fiber. Curing takes place in the mold. The fiber can be woven or braided into an approximation of the final shape, or preform, before being placed into the mold.

\section{Filament Winding}

Filament winding can produce very large shapes, provided that the curvature is everywhere positive. A filament winding machine consists of a rotating mandrel and a fiber dispensing head that travels the length of the mandrel. Synchronized with the mandrel rotation, the head can change the angle of the fiber with respect to the mandrel axis, so that helical plies optimized to handle expected loads can be laid down.

These machines are programmable, so that different winding patterns can be specified. The mandrel can also be changed, allowing even more flexibility. 


\section{DISCLAIMER}

This report was prepared as an account of work sponsored by an agency of the United States Government. Neither the United States Government nor any agency thereof, nor any of their employees, makes any warranty, express or implied, or assumes any legal liability or responsibility for the accuracy, completeness, or usefulness of any information, apparatus, product, or process disclosed, or represents that its use would not infringe privately owned rights. Reference herein to any specific commercial product, process, or service by trade name, trademark, manufacturer, or otherwise does not necessarily constitute or imply its endorsement, recommendation, or favoring by the United States Government or any agency thereof. The views and opinions of authors expressed herein do not necessarily state or reflect those of the United States Government or any agency thereof. 
DISCLAMMER

Portions of this document may be illegible in electronic image products. Images are produced from the best available original doccument. 
The three main constraints imposed by the filament winding process, in order of increasing difficulty to overcome, are:

1. The mandrel must be removed from the interior of the complete structure.

2. The object must have positive curvature everywhere.

3. The mandrel must be fabricated. If the shape does not allow the mandrel to be removed intact (e.g., a tube with wider diameter in the middle than at the ends), it can be removed destructively. Plaster and salt mandrels have been used for this purpose.

An object with reentrant curvature can be filament wound, if the winding is followed by suitable postprocessing, such as hand layup. But the requirement for a mandrel cannot be avoided.

\section{Fiber Placement}

This technique was described above. It is an improvement on filament winding in that it can produce shapes with reentrant curvature. However, it still requires a mandrel, and therefore retains the associated disadvantages. Furthermore, the cost of fiber placement machines is very high.

\section{Pultrusion}

Pultrusion is the most economical fabrication method for abjects having constant cross-section. The fiber and uncured resin are pulled through a heated die which simultaneously shapes and cures the product. Beams and driveshafts are examples of products wellsuited to this process.

Note that while this process does not require a mold, a die of the cross-sectional shape is needed.

\section{Braiding and Weaving}

This technique was mentioned in the discussion of RTM. Dry fibers are braided or woven into configurations optimized for the expected load, and approximately the shape of the target. The resulting network is placed in a mold, impregnated with resin, and cured. The braiding and weaving is done by programmable machines.

Cost savings of up to $50 \%$ have been observed compared to filament winding. A wide range of shapes can be produced with the same equipment, and smooth transitions from one shape to another are possible. A mold is still required for resin impregnation and curing.

\section{SYSTEM DESCRIPTION}

Our system consists of two main components. The first is a reconfigurable forming surface on which heat and pressure are applied to the composite material. This is used to mold and cure each patch of the final shape. The second component is a 6 DOF robot arm, whose role is to position the evolving structure so that the patch currently being molded is held at the proper pose. Both the forming apparatus and the robot are controlled by a single controller.

Figure 1 is an overall view of the system.

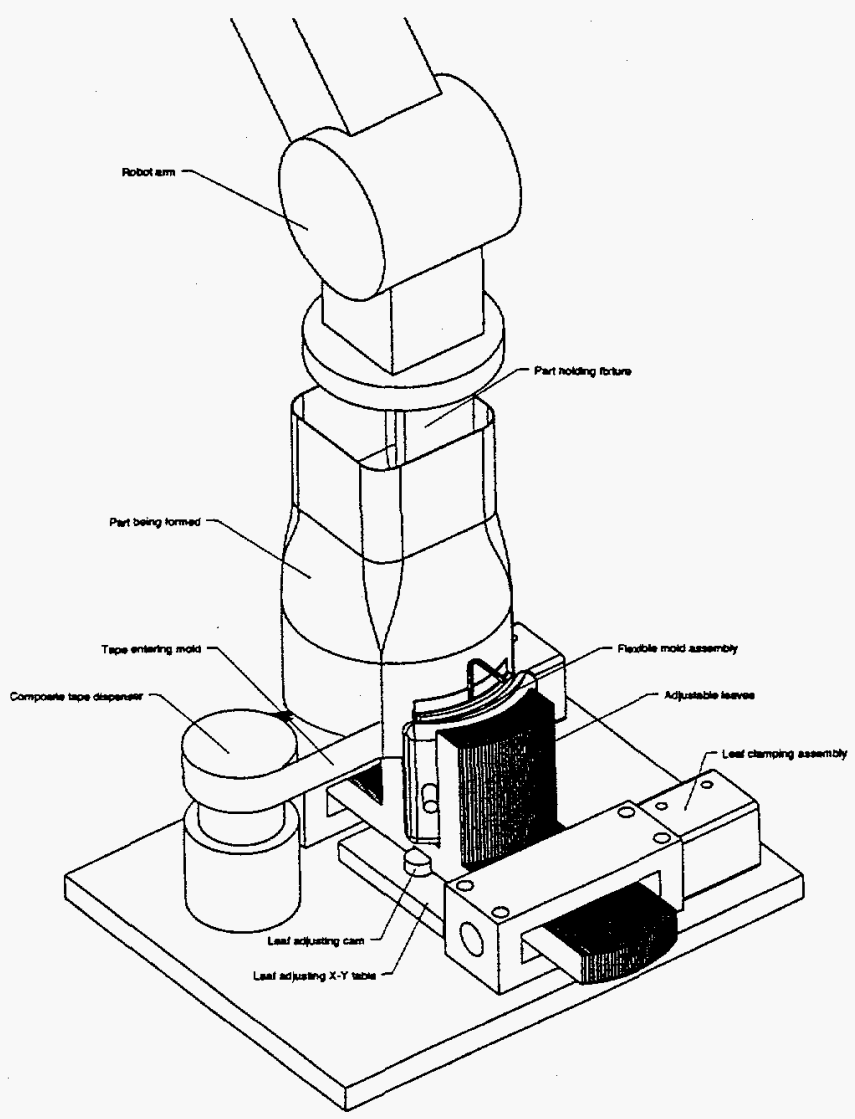

Figure 1: Overall view of agile composite forming apparatus

The attachment of the robot to the part is surprisingly simple. The arm simply grasps the part holding fixture. The part-holding fixture can be any piece of material attached directly to the part being made. The attachment is made by inserting the part-holding fixture into the forming system along with the composite material, so that the first patch is fused directly to the fixture. The cross-section of the portion of the part fused to the fixture is cut off when the part is complete (similar to a sprue in molding). Because the shape of the patch and the location and orientation of the robot relative to the formed patch are continuously variable, very few limitations exist on the shape of the part that can be produced. 
The original system used a Fanuc RJ controller and S-800 arm. Both commingled and preconsolidated composites of thermoplastic resin and continuous fiber material have been successfully formed with this apparatus. We have since implemented it with a Stäubli arm and Adept MV controller.

We now describe the form and function of this forming system, then how it is integrated with the robot. Figure 2 shows a cross section of the forming system. The mold is shaped by a number of adjustable leaves that act like a contour gauge. By adjusting the position of the leaves, a large class of continuous twodimensional curves can be realized. In the current system, the total length of the forming surface composed of the edges of the leaves is about two inches. The required patch shape is made by the leaf-adjusting cam, which runs through the slot on the bottom of the apparatus. The cam is driven by an $\mathrm{X}-\mathrm{Y}$ table, commanded to move in a series of paths that result in the required shape. The commands to the $X-Y$ table are sent from the controller to the $\mathrm{X}-\mathrm{Y}$ motors on a serial line. Once the leaves are in the proper position, the clamping assembly is actuated to hold the leaves in place during the forming process. The patch is then molded and cured by applying heat and pressure to the portion of the part between the leaves.

Pressure is applied by inflating the silicone rubber bladder (on the right in figure 2), and heat is applied by the flexible heater that is folded so that both sides of the part are heated. A thermocouple on the back side of the heater is used to switch current to the heater. The Teflon separator serves as the mold release, and the alumina felt insulates the heated section from the forming leaves. It also smooths out the stairstepping that the leaves introduce. The pnuematics for the bladder and the electronics for the heater and thermocouple are controlled using the robot controller I/O system.

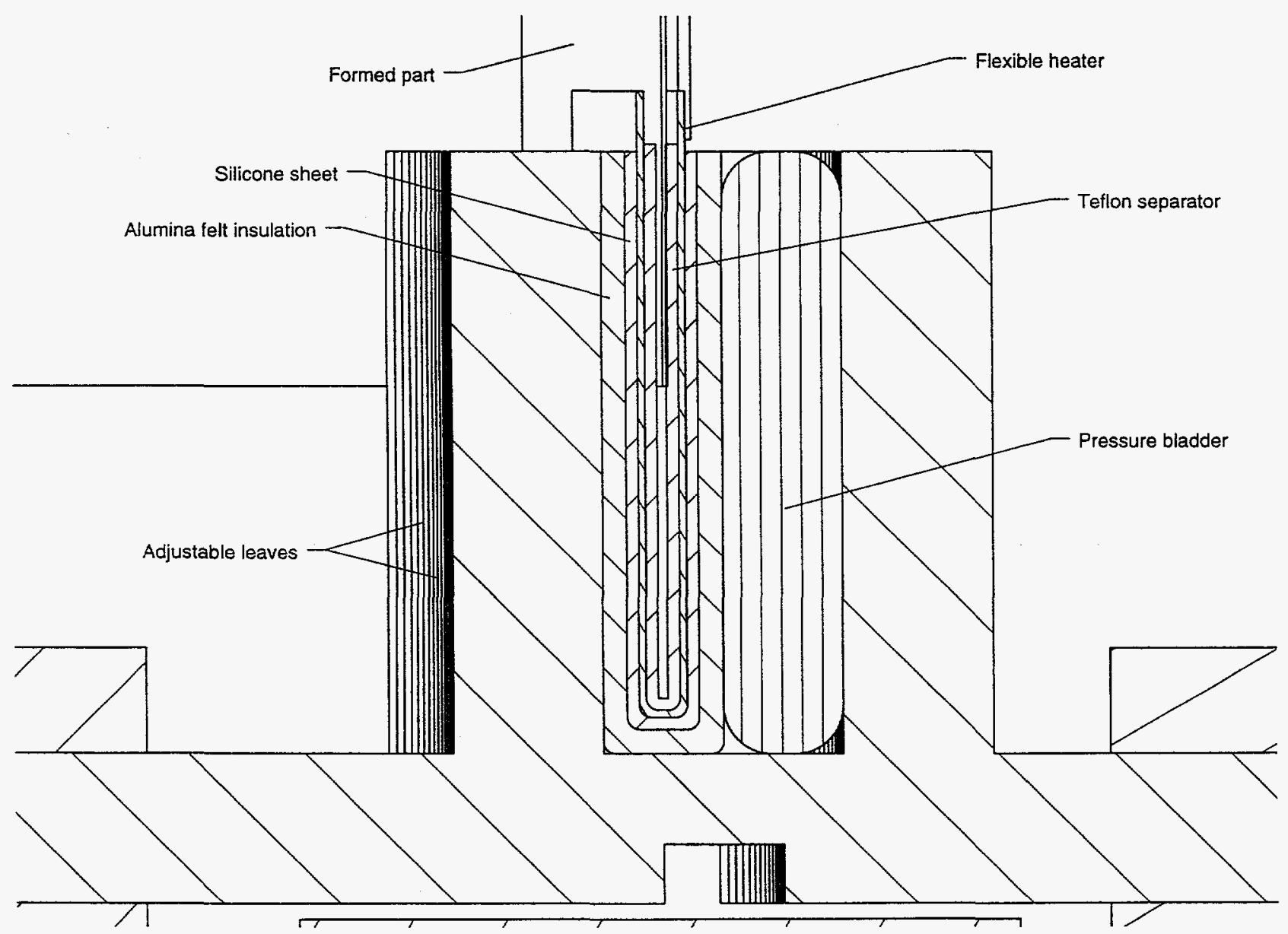

Figure 2: Cross section of flexible mold assembly 
Next, we describe system control. Figure 3 is a control schematic.

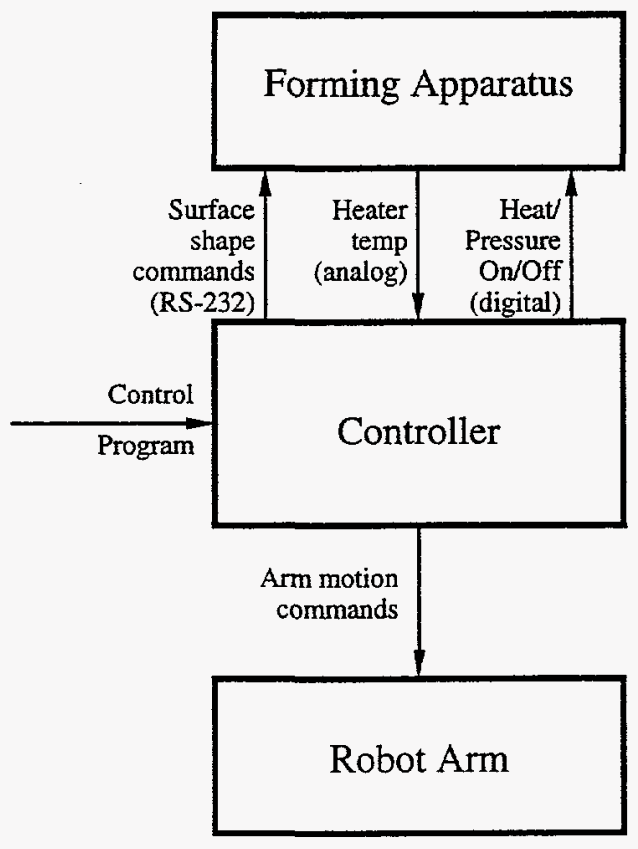

Figure 3: System Block Diagram

The following control cycle is executed repeatedly until the shape is complete. Initially, the heater is off, the bladder is vented, and the clamps are not set.

1. Configure the forming surface, and clamp.

2. Move robot arm so that the next patch to form is at the correct pose (steps 1 and 2 may need to be performed concurrently).

3. Inflate bladder and turn on heater.

4. When heater reaches temperature appropriate for material, switch off.

5. When heater reaches material consolidation temperature, vent bladder.

We have found that the bladder need only be inflated to $3 \mathrm{psi}$. This pressure is surprisingly low, since initial tests with hydraulic presses had indicated that 50-100 psi would be necessary. Such pressures are also needed in many conventional composite layup systems. However, we have observed that pressures greater than 3 psi lead to distortion of the fibers and flashing of the resin. Furthermore, full consolidation is obtained at this pressure. We suspect that the low pressure suffices because it is hydrostatic, whereas presses do not provide true hydrostatic pressure.

\section{An Example}

This section describes how the system is programmed to produce a right circular cylinder. The winding pattern required to realize the desired shape must be specified. A helix having radius $a$ and pitch $c$ is taken as this winding pattern; $c$ is chosen to provide the right amount of overlap on successive wraps. The parametric representation of the helix is:

$$
a \cos (s / w) \mathbf{i}+a \sin (s / w) \mathbf{j}-c(s / w) \mathbf{k}
$$

where $w=\sqrt{a^{2}+c^{2}} ; s / w$ serves as the arc length parameter for the cylinder. Arc length corresponds directly to the amount of composite material paid out in forming the cylinder. That is, the number of steps of the forming process stands in for the arc length of the winding pattern.

For each step, we must determine the shape of the forming surface and the pose of the robot which holds the structure built so far. For the cylinder, the forming surface shape is not changed; it is the arc of a circle of the desired radius. The pose of the robot traces out the helix given as the winding pattern. The pose at each step is easily determined, as follows.

For space curves satisfying certain continuity, differentiability, and curvature conditions, such as the helix, there is a unique set of three mutually orthogonal unit vectors at each point on the curve (Kreyszig, 1972). These vectors are called the unit trihedron of the curve at the given point, and serve as the specification of the orientation of the robot for that step of the process. The unit trihedron consists of the unit tangent vector, the unit principal normal vector, and the unit binormal vector. Let $r(s)$ be the position vector of the curve; then $\dot{\mathbf{r}}(s)$ is the tangent vector (the dot indicates differentiation with respect to the parameter $s$ ). If the parameter is the arc length, then the tangent vector is guaranteed to be of unit magnitude. We denote the unit tangent vector by the symbol $\mathbf{u}(s)$; like the other members of the unit trihedron, its value is functionally dependent on the parameter.

The principal normal is defined as $\ddot{\mathbf{r}}(s)$; its magnitude is defined to be the curvature of the curve. Therefore, the unit principal normal is obtained from the principal normal by dividing it by the curvature of the curve at that point (which cannot be zero). The unit principal normal vector is denoted by $\mathbf{p}(s)$.

The unit binormal $\mathbf{b}(s)$ is defined as

$$
\mathbf{b}(s)=\mathbf{u}(s) \times \mathbf{p}(s) .
$$


The procedure used to obtain a program to trace the helix is to compute the unit trihedron as a function of the arc length, and use it to determine the required pose for each step of the forming process. The unit trihedron is represented as a rotation matrix. The Fanuc R-J controller requires that poses be specified as yawpitch-roll triples, so we had to take the further step of extracting these values from the rotation matrix. The procedure given on page 47 of (Craig, 1989) can be used for this.

In particular, the helix required for the coordinate system used in our workcell is given parametrically as

$$
\mathbf{r}(s)=-a \cos (s / w) \mathbf{i}-a \sin (s / w) \mathbf{j}-c(s / w) \mathbf{k},
$$

leading to the rotation matrix

$$
\left(\begin{array}{ccc}
\cos (s / w) & \sin (s / w) & 0 \\
(a / w) \sin (s / w) & -(a / w) \cos (s / w) & -c / w \\
(c / w) \sin (s / w) & -(c / w) \cos (s / w) & -a / w
\end{array}\right) .
$$

The first row is $\mathbf{p}(s)$, the second is $\mathbf{u}(s)$, and the third is $\mathbf{b}(s)$. Then, when the parameter is zero, the first row projected onto the $x y$ plane is parallel to the $x$ axis, and the second row projected onto the $x y$ plane is parallel to the $y$ axis.

Finally, the yaw-pitch-roll angles required are extracted using the following formulas (where $\beta=$ $\left.\left[((a / w) \sin (s / w))^{2}+(\cos (s / w))^{2}\right]^{1 / 2}\right)$ :

$$
\begin{gathered}
\text { yaw }=\operatorname{atan} 2\left(\frac{-(c / w) \cos (s / w)}{\beta}, \frac{-(a / w)}{\beta}\right) \\
\text { pitch }=\operatorname{atan} 2\left(\frac{-(c / w) \sin (s / w)}{\beta}, \beta\right)
\end{gathered}
$$

and

$$
\text { roll }=\operatorname{atan} 2\left(\frac{(a / w) \sin (s / w)}{\beta}, \frac{\cos (s / w)}{\beta}\right) .
$$

The right circular cylinder illustrated here affords a simple closed form solution. In general, complex parts will be produced using an identical analysis but employing numerical solutions of the equations.

\section{CONCLUSION}

We have described a novel rapid prototyping method that produces structures made of continuous fiber polymer-matrix composites, but does not use a mold. We have implemented a prototype, and demonstrated its feasibility, using commingled and preconsolidated thermoplastic and graphite composite material. We have produced cylindrical objects under automatic control using this system. Producing non-convex shapes with the system is obviously possible, simply by reconfiguring the forming surface as appropriate.

Because our system consists of programmable devices ( $a$ robot arm, and an X-Y stage connected to the reconfigurable forming surface), the control program needed to produce a particular shape can be automatically generated from an electronic model of the shape. We are currently working on this.

\section{REFERENCES}

Craig, J. J., 1989, Introduction to Robotics: Mechanics and Control, Addison-Wesley, Reading, Massachusetts.

Harper, C. A. (ed.), 1992, Handbook of Plastics, Elastomers, and Composites, 2nd edition, McGraw-Hill, New York, chapter 5.

Kreyszig, E., 1972, Advanced Engineering Mathematics, 3rd edition, John Wiley and Sons, New York.

Olsen, H. B., and Craig, J. J., 1993, "Automated Composite Tape Lay-Up Using Robotic Devices", Proceedings, 1993 IEEE International Conference on Robotics and Automation, IEEE Computer Society Press, Los Alamitos, CA, Vol. 3, pp. 291-297. 